\title{
EULER ALGEBRA*
}

BY

E. T. BELL

\section{Preliminary}

1. Introduction. The majority of arithmetical functions specify either additive or multiplicative properties of numbers, ordinary or algebraic. For additive functions the appropriate analysis is the theory of integral power series; for multiplicative, the theory of Dirichlet series and Euler products. Of the last the simplest example is $\Pi\left(1-p^{-s}\right),|s| \geqq 1$, extending to all primes $p>1$. A relation between arithmetical functions which can be so interpreted that it remains true when the arguments of all the functions are replaced by suitably defined elements other than numbers is called qualitative. Such relations are numerous. A simple example is: the totient of an integer is equal to the sum of the totients of all the divisors of the integer. A nonqualitative relation is called quantitative. In the derivation of either kind of relation infinite processes may or may not be used. If the former, then in the case of quantitative relations the convergence of the processes is essential, while in the case of qualitative relations it is irrelevant. Milder instances of the last assertion are sometimes said to be self-evidently true, in particular this: the results obtained by equating coefficients after formal manipulations of series are obviously valid, and it is unnecessary to examine the convergence of the series. Whether obvious or not the general assertion can be proved and is established incidentally in the course of this discussion.

In the investigation of qualitative relations an algebra $E$ (Euler) emerges as the appropriate algorithm. The elements upon which $E$ is ultimately based are abstract, that is, any marks subject to the formal laws of common algebra. This algebra $E$ is in a sense the resultant of two much simpler algebras, $C$ (Cauchy) and $D$ (Dirichlet). $C$ is the algebra of power series in one variable, and is equivalent to the Grassmann-Gibbs indeterminate-product algebra generated by a modulus and a single unit; $D$ is the algebra of Dirichlet series and is the direct product of an infinite number of algebras $C . C$ and $D$ are necessary preliminaries for $E$. By means of $E$ the qualitative properties above described receive a wide generalization. Numerous applications of $E$ have

\footnotetext{
* Presented to the Society, San Francisco Section, April 7, 1923.
} 
been made, both to unify existing treatments of arithmetical functions and to obtain new results in profusion. For simplicity, and to preserve the continuity of the discussion, we omit illustrations and applications except where necessary to make clearer the nature of the abstract theory. For easy reference we put here some definitions upon which is based everything that follows.

(1) If the sum, difference, product and quotient (the divisor not being any one of certain elements, called excepted) of any two identical or distinct elements of a set is an element of the set, the set is called a system.

A system thus differs from an abstract field only by the exclusion of division by each of several elements instead of by one. It is assumed that each rational combination formed in accordance with (1) has a unique interpretation, and that there is at least one set of interpretations of all such combinations which is self-consistent.

(2) If with respect to the elements of a given system a rational operation is unspecified beyond the condition that it obeys the formal laws of common algebra, the operation is called formal, otherwise, special. A system is abstract or special according as none or at least one of the four rational operations is special.

For example, if the elements $a, b, \ldots$ of the system $S$ are the ideals of a realm $R$, the combinations $a \pm b$ (not to be confused with $(a+b),(a-b)$, the G. C. D. and L. C. M. of $a, b$ ) are formal, for the sum $a+b$ and the difference $a-b$ of two ideals are purely formal. That no interpretations in either $S$ or $R$ beyond the formal need be assigned $a \pm b$ to obtain results of importance in $S, R$ is evident from established usage.

(3) The unit $\varepsilon$ with respect to formal multiplication has the properties $\varepsilon f=f=f \varepsilon, f$ any element of the system.

(4) Division by $\varepsilon$, multiplication, addition and subtraction being as in (1), the aggregate of elements derived by iterations of these operations from a given set of elements is called an annulus, which is abstract or special according as none or some of the above operations are special, and the set is its base.

An annulus thus differs from a system by the omission of division except by the multiplicative unit. Theorems valid for an annulus have therefore a greater extension than their analogues for a system. If the elements are algebraic numbers and the rational operations as in arithnetic, the definition of annulus becomes that of Ring (German), anneau (French).

(5) If some or all of the elements of a system (or annulus) can be arranged in a definite order by some rule, the arrangement is called a sequence. An element may occur more than once in a sequence.

(6) A sequence whose first element is zero is called exceptional.

(7) The notation $(a)_{n}$ is used for the sequence $a_{0}, a_{1}, a_{2}, \cdots, a_{n}, \ldots$ in which the suffixes of the successive elements run $0,1,2, \cdots, n, \cdots$, and the notation $\{a\}_{n}$ for the sequence $a_{1}, a_{2}, \cdots, a_{n}, \cdots$ in which the suffixes run 
$1,2, \cdots, n, \cdots$ By an obvious change in the notation of the elements any sequence may be written in either notation. It will be of assistance to observe that the $(a)_{n}$ notation is used exclusively with what are called $C$ processes, the $\{a\}_{n}$ with $D$.

(8) In $C, D$ the excepted elements are the exceptional sequences. In other words, division as defined presently for sequences in either algebra $C, D$ is possible when and only when the divisor is a sequence whose first term is different from zero. This actually is a theorem rather than a definition, but we state it here for emphasis and clearness. Its proof is immediately obvious from the definitions of division in $C, D$.

(9) Two sequences $(a)_{n},(b)_{n}$ are equal, $(a)_{n}=(b)_{n}$, when and only when $a_{i}=b_{i}(i=0,1,2, \cdots)$; and $\{a\}_{n}=\{b\}_{n}$ when and only when $a_{i}=b_{i}$ $(i=1,2, \cdots)$.

From the definition it will appear later that we might have taken instead of sequences as the elements from which to construct $C, D, E$, certain hypercomplex numbers.

It will not be necessary hereafter to emphasize that rational operations indicated by the usual notations of common algebra are formal as in (2) unless otherwise stated, for where they are special they are so indicated by the appropriate symbolism. The last is necessary in order to prevent confusion in reading formulas, for in all four distinct sets of meanings (including the formal) are assigned to the operations.

In all that follows it is assumed that the $a, b, c, \cdots$ with any suffixes are elements of some system. All proofs in the paper depend only upon the foregoing definitions, of which (9) is the most frequently used. For certain developments it is advantageous, however, to replace (9) by (9.2), which comes from the following fundamental postulate (or definition):

(9.1) The series $\sum a_{n} \equiv a_{0}+a_{1}+\cdots+a_{n}+\cdots$ is uniquely deternined by the sequence $(a)_{n}$ of its terms; or, a series is uniquely determined by the sequence of its terms.

We assert only that when the law of formation of the successive elements $a_{0}, a_{1}, \cdots, a_{n}, \cdots$ of the sequence is assigned, the terms, and in particular the general term, of the series are uniquely known. The indicated sum $a_{0}+a_{1}+\cdots+a_{n}+\cdots$ is merely the symbol obtained by writing the elements of the sequence in their given order with plus signs between them. In particular, if the elements $a_{0}, a_{1}$, are numbers and addition is as in arithmetic it is immaterial under the definition (9.1) whether or not a "sum" of the series exists; if the elements are not numbers, "sum" is meaningless, "sum" here being understood in its customary sense in relation to series, viz., as one definite number which may consistently replace the series in numerical calculations. This sufficiently emphasizes that everywhere in this paper "series" has the meaning (9.1) and no other.

The symbolic equality $(a)_{n} \sim \sum a_{n}$ is to be real: the series determined by $(a)_{n}$ is $\sum a_{n}$; and we shall say that each of $(a)_{n}, \sum a_{n}$ is associated with the other. 
(9.2) Two series $\sum a_{n}, \sum b_{n}$ are defined to be equal, $\sum a_{n}=\sum b_{n}$, when and only when their associates are equal, $(a)_{n} \sim \sum a_{n},(b)_{n} \sim \sum b_{n},(a)_{n}=(b)_{n}$.

Note that under this definition if the terms $c_{n}$ are numbers and addition is as in arithmetic, and if $\sum c_{n}^{\prime}$ is the series obtained by rearrangement of terms from the absolutely convergent series $\sum c_{n}$, we do not have $\sum c_{n}=\sum c_{n}^{\prime}$.

(9.3) As usual in mathematical logic two relations are called formally equivalent if each implies the other.

The connections between the operations of the algebras $C, D, E$ with operations upon series will be pointed out incidentally in the development of each algebra. The consequences of operations $X(X=C, D, E)$ will be shown to be formally equivalent to the consequences of certain other operations upon series. Hence if we establish the validity of the first consequences, that of the second will follow, and as a convenience in manipulation we may when desired replace the first set of operations by the second.

It will be well here to give some indication of the origin of the following abstract theory which, although it has an interest as an example of an algebra isomorphic (except for division by more than one "zero") to common algebra yet widely different in content, is important chiefly for the uses to which it has been put. There exists a great mass of special theorems concerning arithmetical functions clustering about the unique factorization theorem. These have been stated and proved individually by various methods, the proofs in some cases, where they depend upon devices peculiar to the theory of numbers, degenerating to a succession of divinations without any unifying principle. In several instances it seems almost as if the results had first been found by means of obvious infinite processes and then translated for esthetic reasons into pure arithmetic. In working over this mass of results it was noticed that it could be unified, made symmetrical by extension in several directions, and finally reduced to an isomorph of common algebra by means of $E$. It will perhaps seem that such simple processes as those of $E$ can lead to nothing less obvious than the relations from which we start. This is not the case. Even so trivial an identity as $\alpha=\alpha \beta / \beta$ when developed in $E$ gives at once numerous valuable consequences, one of which is the totient theorem already cited, upon choosing for $\alpha, \beta$ the simplest rational functions of one or two variables $t, \xi$, such as $1 \pm \xi, 1 \pm \xi^{2}, 1 \pm t \xi, 1 \pm t^{2} \xi^{2}$, etc. Many of these consequences have formed the subjects of notes or elaborate papers, summarized in volume 1, chapters V, X, XIX of Dickson's History of the Theory of Numbers. When the appropriate elementary algebra is used, the arithmetical relation often becomes as obvious as $\alpha=\alpha \beta / \beta$. One important application is outlined in $\S 12$; many others, in complete detail, will be found in the paper referred to in $\S 11$, footnote.

2. Algebra $C$. The $C$ sum $(a+b)_{n}$ of $(a)_{n}$ and $(b)_{n}$ is the sequence $a_{i}+b_{i}$ $(i=0,1, \cdots)$; the $C$ difference $(a-b)_{n}$ is the sequence $a_{i}-b_{i}(i=0,1, \ldots)$ 
and the $C$ zero sequence $(0)_{n}$ is $\left(a_{i}-a_{i}\right),(a)_{n}$ being any sequence. Obviously $(a+b)_{n}=(b+a)_{n}$, and if $(a+b)_{n}=(c)_{n},(b+d)_{n}=(e)_{n}$, then $(c+d)_{n}$ $=(a+e)_{n}=(a+b+d)_{n}=a_{i}+b_{i}+d_{i}(i=0,1, \cdots)$. Hence $C$ addition of sequence is commutative and associative, and similarly for subtraction. We shall denote the $n$th element $a_{n}+b_{n}+d_{n}$ of $(a+b+d)_{n}$ by $n(a+b+d)$, and so in all like cases. Thus the $n$th element of $(a, b)_{n}$ next defined is $n(a, b)$. The $C$ product $(a, b)_{n}$ of $(a)_{n},(b)_{n}$ is the sequence $n(a, b)(n=0,1, \cdots)$, where ${ }_{n}(a, b)=a_{n} b_{0}+a_{n-1} b_{1}+\cdots+a_{1} b_{n-1}+a_{0} b_{n}$.

Proceeding similarly with $(a)_{n},(b)_{n}, \cdots,(k)_{n}$ we obtain their $C$ product $(a, b, \cdots, k)_{n}$ defined by $n(a, b, \cdots, k)=\sum a_{\alpha} b_{\beta} \cdots k_{x}, \alpha+\beta+\cdots+x=n$, $0 \leqq \alpha, \beta, \cdots, x \leqq n$. The unit $(u)_{n}$ with respect to $C$ multiplication is the sequence $u_{0}=\varepsilon$ (cf. $\left.\S 1(3)\right), u_{j}=0, j>0$. Obviously $(a+b, c)_{n}=(a, c)_{n}$ $+(b, c)_{n}$, etc., and $C$ multiplication is commutative, associative, and with respect to $C$ addition and subtraction of sequences, distributive.

THEOREM I. Any set of sequences, formed from the elements of a system, is the base of a special annulus in which the rational operations are $C$ addition, subtraction and multiplication of sequences, and all the elements of the annulus are sequences.

This is the $C$ amuulus to the given base. The number of elements (sequences) in the base may be either finite or infinite.

To connect this with series let $t$ be a parameter which by definition has with respect to $a_{b}, b_{j}, c_{k}, \cdots$ all the formal properties of common algebra, so that $t^{n} a_{i}=a_{i} t^{n}$, etc. Beyond this $t^{n} a_{i}$ is undefined. For all integral values $\geqq 0$ of $n$ write $a_{n}=a_{n} t^{n}, \beta_{n}=b_{n} t^{n}, \cdots, \gamma_{n}$ $=c_{n} t^{n}$, and put, according to (11),

$$
(\alpha)_{n} \sim \sum a_{n} t^{n}, \quad(\beta)_{n} \sim \sum b_{n} t^{n}, \quad \cdots, \quad(\gamma)_{n} \sim \sum c_{n} t^{n} .
$$

Then from Theorem $I$ it follows that for a definite choice of the signs, $( \pm \alpha \pm \beta \pm \cdots \pm \gamma)$ $\sim\left( \pm a_{n} \pm b_{n} \pm \cdots \pm c_{n}\right) t^{n}, \quad \sum_{n}(a, b, \cdots, c) t^{n} \sim(\alpha, \beta, \cdots, \gamma)_{n} \sim \sum a_{n} t^{n} \times \sum b_{n} t^{n} \times \cdots$ $\times \sum c_{n} t^{n}$, where, as in all similar cases, the indicated operations upon the series are to be performed formally and the result is then to be rearranged as a power series in $t$.

If $(a)_{n}$ is not an exceptional sequence $(\S 1(6))$, and $\left(a, a^{\prime}\right)_{n}=(u)_{n},\left(a^{\prime}\right)_{n}$ is called the $C$ reciprocal of $(a)_{n}$, and we write $\left(a^{\prime}\right)_{n}=(u / a)_{n}$. From the given relation, $0\left(a, a^{\prime}\right)=\varepsilon, n\left(a, a^{\prime}\right)=0(n>0)$, and hence $a_{n}^{\prime} a_{0}+a_{n-1}^{\prime} a_{1}+\cdots$ $+a_{1}^{\prime} a_{n-1}+a_{0}^{\prime} a_{n}=0(n>0)$; whence, with $a_{0}^{\prime} a_{0}=\varepsilon$ we obtain the explicit form for $n>0$,

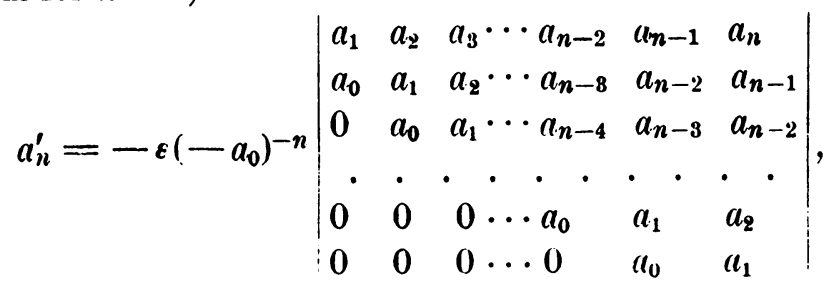


which with $a_{0}^{\prime}=\varepsilon / a_{0}$ completely defines $\left(a^{\prime}\right)_{n}$. It has been sufficiently emphasized that the divisor $(a)_{n}$ in $(u / a)_{n}$ is never an exceptional sequence. Evidently the $C$ product of $(d, u / b)_{n}$ and $(b)_{n}$ is $(d)_{n}$. Hence if $(a)_{n}=(d, u / b)_{n}$, then $(a, b)_{n}=(d)_{n} ;$ and conversely, since the $C$ product of $(a, b)_{n}$ and $(u / b)_{n}$ is $(a)_{n}$, the equality $(a, b)_{n}=(d)_{n}$ implies $(a)_{n}=(d, u / b)_{n}$. Continuing thus, or directly from this result and Theorem I, we infer that, none of $(a),(b)$, $\cdots,(c)$ being exceptional the relation $(a, b, \cdots, c, d, e, \cdots, f)_{n}=(k, l, \cdots, s)_{n}$ implies $\left(d, e, \cdots, f^{\prime}\right)_{n}=(k, l, \cdots, s, u / a, u / b, \cdots, u / c)_{n}$, or as we shall write it more suggestively $(a, e, \cdots, f)_{n}=\left(\frac{l k, l, \cdots, s}{a, b, \cdots, c}\right)_{n}$.

THEOREM II. The aggregate of all sequences formed fiom the elements of a system constitutes a special system in which the four rational operations are $C$ addition, subtraction, multiplication and division, and the excepted elements are the exceptional sequences.

The complicated determinant forms occurring in division are not required in practice; their only use is in demonstrating the existence of $C$ reciprocals. The like applies to $D$. The series equivalent of $C$ division being evident it neel not be written.

By (9) the equality of two sequences is formally equivalent to the identity of their general terms, and Theorem II shows how identities of this kind may be obtained on starting from any given set of sequences. From the foregoing it is clear that the same identities would be reached if we operated with the associated series instead of the sequences as summarized in the following theorem. Hence (cf. the remarks after (9.3)) the formal use of the series is validated.

THEOREM II'. If $R(x, y, \cdots, z)=0$ is a rational velation in any system, and if, further, in algebra $C$ the $C$ relation $R\left((\alpha)_{n},(\beta)_{n}, \cdots,(\gamma)_{n}\right)=0$ holds, where $(\alpha)_{n} \sim \sum a_{n} t^{n},(\beta)_{n} \sim \sum b_{n} t^{n}, \cdots,(\gamma)_{n} \sim \sum c_{n} t^{n}$, then the $C$ relation is equivalent to $R\left((a)_{n},(b)_{n}, \ldots,(c)_{n}\right)=0$, which in turn is formally equivalent to $R\left(\sum a_{n} t^{n}, \sum b_{n} t^{n}, \ldots, \sum c_{n} t^{n}\right)=0$ in the system and is obtained firom the last by equating to zero the coefficient of $t^{n}$.

An alternative point of view may be noted in passing. Let $\varepsilon_{n}$ denote the sequence whose $(n+1)$ th element is 1 , and whose remaining terms are zeros. Then $(a)_{n}$ is $\sum_{0}^{\infty} a_{n} \varepsilon_{n}$, which (as in hypercomplex numbers) may be written $a$. Then algebra $C$ is the Grassmann-Gibbs product algebra generated by a modulus (1) and a single unit $e,\left(\varepsilon_{0}=1, \varepsilon_{n}=e^{n}\right)$. This algebra is equivalent to that of power series in one variable.

As they are frequently useful we consider briefly the meanings to be assigned to irrational operations in $C$. Algebras $D, E$ are similarly discussed in this respect with a few obvious changes in notation and consequent modifications in the interpretations of results, so it will be unnecessary to repeat 
the argument for $D, E$. We are then to assign selfconsistent meanings to irrational functions of the elements, which are sequences, in $C$ such that these are compatible with the rational functions.

If the $a$ in $(a, a, \cdots, a)_{n}$ occurs precisely $r$ times we write this $C$ product $\left(a^{r}\right)_{n}$ and define it to be the $r$ th power of $(a)_{n}$. Then if $(b)_{n}=\left(a^{r}\right)_{n}$ we call $(a)_{n}$ the $r$ th root of $(b)_{n}$ and write $(a)_{n}=\left(b^{1 / r}\right)_{n}$. From this $\left(b^{s / r}\right)_{n}$ is defined for $r, s$ integers $>0$ by $\left(b^{s / r}\right)_{n}=\left(a^{s}\right)_{n}$. The $C$ reciprocal $(u / a, u / a, \cdots, u / a)_{n}$ of $\left(a^{r}\right)_{n}$ is written $\left(a^{-r}\right)_{n}$. Hence from the foregoing $\left(b^{-s / r}\right)_{n}$ is defined and is equal to $\left(a^{-s}\right)_{n}$, the notation being as above. Hence, $t$ being any rational non-zero number, $\left(a^{t}\right)$ is defined. The explicit forms of the elements in this sequence can be written easily, but as they are not required in applications we omit them. Incidentally the explicit forms prove the existence of such sequences. For fractional values of $t$ they enter only as links in chains of transformations. It is readily seen from the definitions that when $t, s, \cdots$ are rational numbers, $\left(a^{t}\right)_{n},\left(a^{s}\right)_{n}, \cdots$ are subject to the formal laws of common algebra.

THEOREM II". Algebra $C$ in its complete form is abstractly identical with common algebra, and hence each identity or theorem in the latter has a unique dual in terms of elements of $C$, and this dual is expressible ultimately as an identity or theorem connecting the nth (general) elements of sequences related to one another by the operations of $C$.

In practice we apply this as if the elements $(a)_{n},(b)_{n}, \cdots$ were those of common algebra, $a, b, \ldots$. Thus we may ignore the special notatious $(a)_{n},(a, b)_{n}$, etc., and write simply $a, b, a b, a / b$, etc. In the results each $a, b \ldots$ is interpreted as the $n$th element of the sequence which it represents, and similarly for $a b$, etc., viz. $a b$ is not the formal product of the $n$th elements of $a, b$, but is the $n$th element of the $C$ product of $a$ and $b$. As remarked, precisely similar considerations apply to $D, E$, and this exposition with a few slight changes can be fitted to them.

We shall reach our goal $E$ through $C$ via $D$. For $E, C$ is fundamental. The point of departure for obtaining relations in $E$ will be seen to be the identities obtained by applying Theorems II, II'. Hence we next indicate the manner in which these may be found. We recall first that in (9.1) any quantitive (numerical) significance of an infinite series was abandoned. Hence we discard also the concept of an analytic function and all of its consequences. Nevertheless we shall assign a meaning to the representation of a function of an element by an infinite series which is significant in all circumstances. In particular if the terms of the series are numbers and the operations as in arithmetic, the function is represented by the series whether or not it is summable by any method.

Let $\alpha, \beta, \cdots, \gamma, x, \lambda, \cdots, \sigma$ be any system of elements, and $(\alpha)_{n},(\beta)_{n}, \cdots$, $(\sigma)_{n}$ sequences as usual. Note that $\alpha$ is not a member of $(\alpha)_{n}$, and similarly for the rest. Let $(\Delta)_{n}$ be a sequence of operators, $\varphi$ any element such that the results of operating with $\Delta_{n}(n=0,1, \ldots)$ upon $\varphi$ are each uniquely significant, and let the result $\Delta_{n} \varphi$ of operating with $\Delta_{n}$ upon $\varphi$ be $\varphi_{n}$, or symbolically $\Delta_{n} \varphi=\varphi_{n}$. We assume that for $m, n$ integers $\geqq 0, \Delta_{m} \varphi_{n}$ 
$=\Delta_{m}\left(\Delta_{n} \varphi\right)=\varphi_{m+n} ;$ so the elements (operators) of $(\Delta)_{n}$ obey with respect to suffixes the index laws of ordinary algebra, thus $\Delta_{m} \Delta_{n}=\Delta_{m+n}=\Delta_{n} \Delta_{m}$, etc. We shall say that $(\varphi)_{n}$ is developed by $(\Delta)_{n}$ from $r$, and write this $(\Delta)_{n} \varphi$ $=(\varphi)_{n}$. When there is no occasion to indicate the specific developer $(\Delta)_{n}$ we shall write $\varphi^{\prime}$ for $(\Delta)_{n} \varphi$. If $\varphi=\psi$, then by definition $\varphi^{\prime}=\psi^{\prime}$. For simplicity we assume that $\Delta_{0} \boldsymbol{y}_{\mathrm{i}} \equiv \varphi_{\varphi_{0}}$ is different from zero. Developers are clissified according to their properties with respect to sums, differences, products and quotients of elements. The $(\Delta)_{n}$ considered now is called, for obvious reasons, a $C$ developer. By definition we take $(\Delta)_{n}(\alpha+\beta+\cdots+\gamma)$ $\equiv(\alpha+\beta+\cdots+\gamma)^{\prime}=\alpha^{\prime}+\beta^{\prime}+\cdots+\gamma^{\prime}$, and this is equal to $(\alpha)_{n}+(\beta)_{n}$ $+\cdots+(\gamma)_{n}$, with a similar definition when some of the signs are negative; $(\Delta)_{n}(\alpha \beta \cdots \gamma) \equiv(\alpha \beta \cdots \gamma)^{\prime}=(\alpha, \beta, \cdots, \gamma)_{n}$, giving the development for a product of elements; and for the development of a quotient

$$
(\Delta)_{n}\left(\frac{\alpha \beta \cdots \gamma}{x \dot{\lambda} \cdots \sigma}\right) \equiv\left(\frac{\alpha \beta \cdots \gamma}{x \lambda \cdots \sigma}\right)^{\prime}=\left(\frac{\alpha, \beta, \cdots, \gamma}{x, i, \cdots, \sigma}\right)_{n}
$$

There can be no confusion if we simplify the writing by putting

$$
(\alpha \beta \cdots \gamma)^{\prime} \equiv \alpha^{\prime} \beta^{\prime} \cdots \gamma^{\prime}, \quad\left(\frac{\alpha \beta \cdots \gamma}{x \lambda \cdots \sigma}\right)^{\prime}=\frac{\alpha^{\prime} \beta^{\prime} \cdots \gamma^{\prime}}{x^{\prime} \lambda^{\prime} \cdots \sigma^{\prime}}
$$

so that the multiplications indicated in the first, $\boldsymbol{\alpha}^{\prime} \boldsymbol{\beta}^{\prime} \cdots \gamma^{\prime}$, are purely symbolic, and likewise for the multiplications $\alpha^{\prime} \beta^{\prime} \cdots \gamma^{\prime}, x^{\prime} \lambda^{\prime} \cdots \sigma^{\prime}$ and the division $\boldsymbol{\alpha}^{\prime} \boldsymbol{\beta}^{\prime} \ldots \boldsymbol{\gamma}^{\prime} / \boldsymbol{\alpha}^{\prime} \hat{\lambda}^{\prime} \ldots \sigma^{\prime}$ in the second.

THEOREM $\mathrm{II}^{\prime \prime \prime}$. The rational relation $R(\alpha, \beta, \cdots, \gamma)=0$ implies $R\left(\boldsymbol{\alpha}^{\prime}, \boldsymbol{\beta}^{\prime}, \cdots, \boldsymbol{\gamma}^{\prime}\right)=0$.

This is an immediate consequence of the definitions and Theorems II, II". A rational relation may in general be written in several distinct forms, say $\alpha \beta+\beta \gamma=\delta$, or $a+\gamma=\beta / \delta$. By the theorem these imply $a^{\prime} \beta^{\prime}+\beta^{\prime} \gamma^{\prime}=\delta^{\prime}, a^{\prime}+\gamma^{\prime}=\delta^{\prime} / \beta^{\prime}$, which are $(\alpha \beta)^{\prime}+(\beta \gamma)^{\prime}=\delta^{\prime}, \alpha^{\prime}+\gamma^{\prime}$ $=(\delta / \beta)^{\prime}$. The accented relation is of the kind in the theorem for algebra $C$, and it has been obtained by development $(\Delta)_{n}$ from the unaccented relation between the elements $\alpha, \beta, \cdots, \gamma$. The extension to irrational relations follows as in Theorem II".

By the definition of $(\Delta)_{n}$ combined with the specific properties of a $C$ developer with relation to sums, etc., it follows that $\Delta_{m}\left(\Delta_{n}(\alpha \beta)\right)$ must be identical with $\Delta_{m+n}(\alpha \beta)$, with the like for sums, differences and quotients, if the definition and specified properties are to be consistent. It therefore remains to show that for at least one class of elements $\alpha, \beta, \cdots, \gamma$ and one developer $(\Delta)_{n}$ these conditions are satisfied. The following is an important example. 
Let the $\alpha, \beta, \cdots, \gamma$ denote single valued functions of $x$ such that all their derivatives exist and are finite and single valued when $x=0$. (Any number other than 0 might be chosen, but the statement is simplest in this case.) Let $\Delta_{n}$ be the operation of taking the $n$th derivative of a given function with respect to $x$, replacing $x$ by 0 in the result, and finally multiplying this by $x^{n} / n$ ! Then so far as sums and differences are concerned it is obvious that $(\Delta)_{n}$ satisfies the conditions in question. The like follows at once for products from Leibniz's theorem for the successive derivatives of a product. Hence the conditions are satisfied also for a quotient.

We have $(\Delta)_{n} \alpha=(\alpha)_{n}$; and if $(\alpha)_{n} \sim \sum \alpha_{n} \equiv \sum \Delta_{n} \alpha$, we call the series $\sum \Delta_{n} \alpha$ the $(\Delta)_{n}$ development of $\alpha$. In the above special example the $(\Delta)_{n}$ development is the Maclaurin series. The $(\Delta)_{n}$ development in every case is unique and significant, and from the foregoing theorems such series can be formally manipulated in any system.

3. Algebra $D$. By the remarks on notation in $\$ 1(7)$, it is unnecessary to repeat the definition and properties of adaition and subtraction for sequences written in the $\{a\}_{n}$ notation. For uniformity with what follows these operations in this case are called $D$ addition and subtraction. The $D$ product of the sequences $\{a\}_{n},\{b\}_{n}, \cdots,\{k\}_{n}$ is the sequence $\{a, b, \cdots, k\}_{n}$ whose $n$th element $n\{a, b, \cdots, k\}$ is defined by

$$
\begin{aligned}
{ }_{n}\{a, b, \cdots, k\} & =\sum a_{\alpha} b_{\beta} \cdots k_{x}, \quad \alpha \beta \cdots x=n, \\
1 & \leqq \alpha, \beta, \cdots, x \leqq n,
\end{aligned}
$$

the notation indicating that the summation extends to all possible sets of positive integers $\alpha, \beta, \cdots, x$ whose product is $n$, the number of integers in each set being equal to the number of sequences whose product is taken. Clearly $\{a, b, \cdots, k\}_{n}$ is invariant under all permutations of $a, b, \cdots, k$. Hence $D$ multiplication is commutative. To see that it is also associative let $\{a\}_{n},\{b\}_{n}, \cdots,\{k\}_{n}$ be distributed in any way into sets such that each sequence occurs once and once only in all the sets; form the $D$ product for all the sequences in each of the sets, and finally take the $D$ product of these products. Then evidently the result is the $D$ product of all the sequences. Similarly it is seen at once that with respect to $D$ addition and subtraction $D$ multiplication is distributive. The unit with respect to this multiplication is the sequence $\{v\}_{n}, v_{1}=\varepsilon, v_{n}=0(n>1)$.

The sequence $\left\{a^{\prime}\right\}_{n}$ satisfying $\left\{a, a^{\prime}\right\}_{n}=\{v\}_{n}$, where $\{a\}_{n}$ is not exceptional, is called the $D$ reciprocal of $\{a\}_{n}$ and is written $\{v / a\}_{n}$. To simplify the printing put for a moment $(i, j)=0$ or $a_{i / j}$ according as $i / j$ is not or is an integer $>0$. Then proceding as in $C$ we find 


$$
a_{n}^{\prime}=(-1)^{n-1} a_{1}^{-n}\left|\begin{array}{ccccc}
(n, n-1) & (n, n-2) & \cdots & (n, 2) & (n, 1) \\
(n-1, n-1) & (n-1, n-2) & \cdots & (n-1,2) & (n-1,1) \\
0 & (n-2, n-2) & \cdots & (n-2,2) & (n-2,1) \\
\ldots & \cdots \cdots & \cdots & \cdots & \cdots \cdots \\
0 & 0 & \cdots & (3,2) & (3,1) \\
0 & 0 & \cdots & (2,2) & (2,1)
\end{array}\right|
$$

which with $a_{1}^{\prime}=\varepsilon / a_{1}$ completely defines $\{v / a\}_{n}$. Continuing, with the necessary and obvious modifications, as in $C$ we infer that the first of the following relations implies the second, none of $\{a\}_{n},\{b\}_{n}, \cdots,\{c\}_{n}$ being exceptional:

$$
\begin{gathered}
\{a, b, \cdots, c, d, e, \cdots, f\}_{n}=\{k, l, \cdots, s\}_{n}, \\
\{d, e, \cdots, f\}_{n}=\{k, l, \cdots, s, v / a, v / b, \cdots, v / c\}_{n},
\end{gathered}
$$

or, as we shall write it,

$$
\left\{d, e, \cdots, f_{n}=\left\{\frac{k, l, \cdots, s}{a, b, \cdots, c}\right\}_{n}\right.
$$

Theorems III, IV, IV". Replace $C$ by $D$ in Theorems J, II, II".

The connection with Dirichlet series is obtained thus. For all integral values $>0$ of $n$ write $a_{n}=a_{n} n^{i}, \beta_{n}=b_{n} n^{i}, \cdots, \gamma_{n}=c_{n} n^{t}$, and put $\{a\}_{n} \sim \sum a_{n} n^{i},\{\beta\}_{n} \sim \sum b_{n} n^{t}, \cdots$, $\{r\}_{n} \sim \sum c_{n} n^{t}$. Then for a definite choice of the signs,

$$
\begin{gathered}
\{\square a \pm \cdots \pm \gamma\}_{n} \sim \sum_{n}\left( \pm a_{n} \pm b_{n} \pm \cdots \pm c_{n}\right) n^{i} \\
{ }_{n}\{a, \beta, \cdots, \gamma\}=\{a, b, \cdots, c\} n^{2}
\end{gathered}
$$

and to find the series associated with $\{\alpha, \beta, \cdots, \gamma\}_{n}$ we multiply together formally the associated series of $\{\alpha\}_{n},\{\beta\}_{n}, \cdots,\{\gamma\}_{n}$ and rearrange the result in the form $\sum k_{n} n^{i}$ :

$$
\{\alpha, \beta, \cdots, \gamma\}_{n} \sim \sum a_{n} n^{\imath} \times \sum b_{n} n^{\imath} \times \cdots \times \sum c_{n} n^{\imath}=\sum k_{n} n^{\imath}
$$

The further development parallels that for algebra $C$ upon replacing the $(a)_{n}$ notation by the $\{a\}_{n}$ and referring the discussion to coefficients of $n^{2}$ instead of $t^{n}$ as before."

Corresponding to the alternative for $C$ we have for $D$ the algebra in which the units $\varepsilon_{n}$ are commutative, with the law of combination $\varepsilon_{m} \varepsilon_{n}=\varepsilon_{m+n}$. This algebra is the direct product of an infinite number of algebras $C$, generated by a modulus and units $e_{1}, e_{2}, \cdots$ respectively. The correspondence between the algebras is as follows. Let $n=\alpha^{a} \beta \cdots$ be the resolution of $n$ into its prime factors, and let $\alpha$ be the $\lambda$ th prime, $\beta$ the $\mu$ th prime, etc. Then $\varepsilon_{n}=\varepsilon_{\lambda}^{a} \varepsilon_{\mu}^{b} \cdots$. 


\section{ALGEBRA $E$}

4. By the proper change in notation any sequence may be written in the form $a_{2}, a_{3}, a_{4}, \cdots, a_{n}, \cdots$, and if we put $a_{1}=\varepsilon(\S 1(3))$ whatever the sequence, we consider throughout this part $\{a\}_{n}$, that is, $a_{1}(=\varepsilon), a_{2}, a_{3}, \cdots$, instead of the original sequence $a_{2}, a_{3}, \cdots$. This inclusion of $\varepsilon$ as the first element in every sequence is merely a device to shorten the statements of processes and results in what follows. We now make a radical change in notation and elaborate the concepts upon which $E$ is constructed.

(10) Having written our initial sequence as above prescribed in the form $\{a\}_{n}$ with first element $\varepsilon$, we next put $a_{1}=x_{1}, a_{2}=x_{2}, a_{3}=x_{3}, a_{4}=x_{5}$, $a_{5}=x_{7}, \cdots, a_{n}=x_{p}, \cdots$, where $p$ is the $n$th prime number $\geqq 1$. We thus obtain $\{a\}_{n}$ in the normal script $x_{1}, x_{2}, x_{3}, x_{5}, x_{7}, x_{11}, x_{13}, x_{17}, \cdots, x_{p}, \cdots$, which is written ' $\{x\}_{p}$ and is called a primary sequence. The first element $x_{1}$ of ${ }^{\prime}\{x\}_{p}$ is $\varepsilon$.

This again is nothing more than an artifice by which the algebra is greatly simplified in expression. In the extension of $E$ processes to multiple sequences (not discussed here) the inherent complexity of the generalized concepts is such that a simplified notation is imperative. The extension of the above device is immediate.

(11) The aggregate $X$ of all formal products $x_{a}^{a} x_{\beta}^{b} \cdots x_{\gamma}^{c}$ of positive integral or zero powers $x_{\alpha}^{a}, x_{\beta}^{b}, \cdots, x_{\gamma}^{c}$ of distinct elements $x_{\alpha}, x_{\beta}, \cdots, x_{\gamma}$ of ' $\{x\}_{p}$ is not a sequence until a law is assigned by which the several products are ordered. We adopt first the convention that each product of this type in which all of the exponents $a, b, \cdots, c$ are zero is identical with $x_{1}(\equiv \varepsilon)$. Since $x_{1} x_{n}=x_{n}$ it follows that ' $\{x\}_{p}$, is contained in $X$, that is, every element of ' $\{x\}_{p}$ is an element of $X$. If now $n=\alpha^{a} \beta^{b} \cdots \gamma^{c}$, where $a, b, \cdots, c$ are all different from zero, is the resolution of $n$ into its prime factors $>1$, we define $x_{n}$ by $x_{n}=x_{\alpha}^{a} x_{\beta}^{b} \cdots x_{\gamma}^{c}$ and form the sequence $\{x\}_{n}^{\prime} \equiv x_{1}, x_{2}, x_{3}, x_{4}, \cdots, x_{n}, \cdots$, called the first derivative of the primary ' $\{x\}_{p}$.

Each product is now placed; for example $x_{2}^{5} x_{7}^{2} x_{11}^{8}=x_{2087008}$. Conversely the resolution of each element of $\{x\}_{n}^{\prime}$ into a product of powers of distinct elements of ' $\{x\}_{p}$ is uniquely known; thus $x_{7805}=x_{3}^{2} x_{5} x_{18}^{2}$. If from $\{x\}_{n}^{\prime}$ we delete all elements whose suffixes are composite there remains ' $\{x\}$.

Now obviously $\{x\}_{n}^{\prime}$ may be written in normal script as a primary sequence, say " $\{x\}_{p}$, and we can take its first derivative " $\{x\}_{n}^{\prime}$, or more briefly $\{x\}_{n}^{\prime \prime}$, which is called the second derivative of $\{x\}_{p}^{\prime}$. Continuing thus we define the $r$ th derivative for $r$ any integer $\geqq 1$. For simplicity we consider here only the case $r=1$.

(12) Let $a, b, \cdots, c, \cdots$ denote an infinity of integers each of which may vary from zero to positive infinity, and let $\alpha, \beta, \cdots, \gamma, \cdots$ denote the primes $>1$ in any order. Denote by any one of the infinity of symbols $f_{0}\left(x_{\delta}\right)$, where 
$\delta>1$ is prime, the unit with respect to multiplication in the abstract annulus $(\S 1(4))$ whose base is the aggregate of elements $f_{a}\left(x_{\alpha}\right), f_{b}\left(x_{\beta}\right), \cdots, f_{c}\left(x_{\gamma}\right), \ldots$ where $a, b, \cdots, c, \cdots$ and $\alpha, \beta, \cdots, \gamma, \cdots$ take all values consistent with their definitions; and let each element of the annulus have a unique significance in terms of elements of ' $\{x\}_{p}$. Then for specific $a$ and $\alpha, f_{a}\left(x_{\alpha}\right)$ is called a primary function of $x_{\alpha}$, and similarly for $f_{b}\left(x_{\beta}\right)$ and $x_{\beta}, \cdots, f_{c}\left(x_{\gamma}\right)$ and $x_{\gamma}, \cdots$; and these primary functions are associated with the elements, $x_{a}^{a}, x_{\beta}^{b}, \cdots, x_{\gamma}^{c}, \ldots$ of $\{x\}_{n}^{\prime}$ respectively. From the definition of the unit, $f_{0}\left(x_{\alpha}\right) f_{b}\left(x_{\beta}\right)=f_{b}\left(x_{\beta}\right)$, $f_{0}\left(x_{\alpha}\right) f_{0}\left(x_{\beta}\right)=f_{0}\left(x_{\alpha}\right)=f_{0}\left(x_{\beta}\right)$, and so on.

(13) The notation being as in (12), so that $\alpha>1$ is prime, the sequence $f_{0}\left(x_{a}\right)$, $f_{1}\left(x_{a}\right), f_{2}\left(x_{a}\right), \cdots, f_{n}\left(x_{a}\right), \cdots$, which we shall write $\left(f\left(x_{a}\right)\right)_{n}$, is called a primary functional sequence.

(14) We next order the products contained in the annulus of (12) into a sequence. Let $n=\alpha^{a} \beta^{b} \ldots \gamma^{c}$ be the resolution of $n>1$, and recall that by (11) the element $x_{n}$ of $\{x\}_{n}^{\prime}$ is $x_{\alpha}^{a} x_{\beta}^{b} \cdots x_{\gamma}^{c}$. With this element we associate the product $f_{a}\left(x_{a}\right) f_{b}\left(x_{\beta}\right) \cdots f_{c}\left(x_{\gamma}\right)$ and denote it by either of $f_{x}(n)$ or $f_{n}(x)$. If $m$ is not prime $f_{n}\left(x_{m}\right)$ is meaningless; also $f_{1}(x)$ is not yet defined. As suggested by $1=\alpha^{0}=\beta^{0}=\cdots=\alpha^{0} \beta^{0}=$ etc., we now identify $f_{1}(x)$ with the unit $f_{0}\left(x_{\alpha}\right)$, $\equiv f_{0}\left(x_{\beta}\right) \cdots \equiv f_{0}\left(x_{\gamma}\right)$, etc., defined in (12). When $n$ is given, the place of $x_{n}$ in $\{x\}_{n}^{\prime}$ is known, and likewise for $f_{n}(x)$ in the sequence $f_{1}(x), f_{2}(x), f_{3}(x), \cdots, f_{n}(x), \cdots$, which we shall denote by $\{f(x)\}_{n}$ and call a derived functional sequence. Obviously each product contained in the annulus occurs once and once only in $\{f(x)\}_{n}$, which also contains all primary functional sequences $\left(f\left(x_{p}\right)\right)_{n}, p$ running through all primes $p>1$.

The $x$ in $f_{z}(n)$ or $f_{n}(x)$ indicates only that the arguments of the several $f_{a}, f_{b}, \cdots, f_{c}$ in the formal product which is denoted by either of these symbols are elements of the sequence $\{x\}_{n}^{\prime}$, and $x$ itself is not one of these arguments, for it is not an element of the sequence. If the sequence were $\{y\}_{n}^{\prime}$ our symbol would be written $f_{n}(y)$ or $f_{y}(n)$. The $x$ in $f_{n}(x)$ is thus purely umbral, and has not the usual significance that $f_{n}(x)$ is a function of $x$. But on the other hand the element $x_{n}$ being uniquely determined when $n$ is assigned, $f_{z}(n)$ is in the ordinary sense a function of the positive non-zero integer $n$.

When several derived functional sequences $\left\{f^{\prime}(x)\right\}_{n},\{g(x)\}_{n}, \cdots$ are being considered together we postulate that $f_{1}(x), g_{1}(x), \cdots$ are identical and that (as in $\S 1(1)$ ) the formal sums, differences and products constructed from elements of all the sequences are uniquely significant.

As an example of the notation, $f_{380}(x)=f_{8}\left(x_{2}\right) f_{2}\left(x_{8}\right) f_{1}\left(x_{6}\right)$, since $360=2^{3} 3^{2} 5$ and hence $x_{800}=x_{2}^{8} x_{8}^{2} x_{5}$.

5. $E$ composition. This process, which is fundamental for $E$, has the formal properties of multiplication and is radically distinct from any in $C, D$, although, as will appear, it combines both $C$ and $D$ multiplication. The derived functional sequence $\{f(x)\}_{n}$ is uniquely determined (§4(14)) by the aggregate 
of primary functional sequences $\left(f\left(x_{p}\right)\right)_{n}$ where $p$ runs through all primes $p>1$. Conversely, all of the sequences in this aggregate are necessary for the determination of $\{f(x)\}_{n}$, and the aggregate defines one and only one derived functional sequence. Let us express this by saying that $\{f(x)\}_{n}$ is the $E$ composite $\left[\left(f\left(x_{p}\right)\right)_{n}\right]$ of all the sequences in the aggregate of primary functional sequences $\left(f\left(x_{p}\right)\right)_{n}$, and let us write this symbolically as follows:

$$
\left[\left(f\left(x_{p}\right)\right)_{n}\right]=\{f(x)\}_{n},
$$

which may be read: the $E$ composite of all the sequences in the aggregate $\left(f\left(x_{p}\right)\right)_{n}$ where $p$ runs through all primes $>1$ is the sequence $\{f(x)\}_{n}$, and this sequence is obtained from the aggregate by $E$ composition.

(15) The $E$ composites $\left[\left(f\left(x_{p}\right)\right)_{n}\right],\left[\left(g\left(x_{p}\right)\right)_{n}\right]$ are defined to be equal, $\left[\left(f\left(x_{p}\right)\right)_{n}\right]=\left[\left(g\left(x_{p}\right)\right)_{n}\right]$, when and only when $\{f(x)\}_{n}=\{g(x)\}_{n}$.

Theorems for $E$ operations are thus restatements in terms of $E$ composites of theorems in a $D$ algebra in which the elements are derived functional sequences, and they follow at once from Theorems III, IV, IV", upon translating them into their equivalents in terms of derived functional sequences. The discussion will be more easily followed from the series equivalents of $E$ composition which we consider next.

6. To obtain the formal equivalents of $E$ operations we require the parameters $t_{n}$ which are defined on replacing $x$ in $\S 4(10),(11)$ by $t$. Thus $n=\alpha^{a} \beta^{b} \cdots \gamma^{c}$ being as before the prime factor resolution of $n$, the element $t_{n}$ of $\{t\}_{n}^{\prime}$ is $t_{\alpha}^{a} t_{\beta}^{b} \cdots t_{\gamma}^{c}, t_{\alpha}^{0}=t_{1}=\varepsilon$ ( $\alpha$ any prime), $t_{1} t_{n}=t_{n}$; and if $n=\alpha^{a}$, $t_{n}=t_{a}^{a}$. With respect to the elements of derived functional sequences $\{f(x)\}_{n}$, $\{g(x)\}_{n}, \cdots, t_{n}$ has all the properties of formal multiplication so that $t_{n} f_{m}(x)$ $=f_{m}(x) t_{n}$, and if $t_{n} f_{m}(x)=t_{n} g_{m}(x)$, then $f_{m}(x)=g_{m}(x)$.

Write for a moment $t_{p}^{n} f_{n}\left(x_{p}\right)=f_{n}^{\prime}\left(x_{p}\right)$. Then $\sum_{0} f_{n}^{\prime}\left(x_{p}\right) \sim\left(f^{\prime}\left(x_{p}\right)\right)_{n}$ $\sim \sum_{0} t_{p}^{n} f_{n}\left(x_{p}\right)$. From the definition of $E$ composition and (15) the sequence $\left\{f^{\prime}(x)\right\}_{n}$ is uniquely determined by the aggregate of sequences $\left(f^{\prime}\left(x_{n}\right)\right)_{n}$, where $p$ runs through all primes $>1$. Hence since $\left\{f^{\prime}(x)\right\}_{n} \sim \sum_{1} f_{n}^{\prime}(x)$, the series $\sum_{1} t_{n} f_{n}(x)$ is uniquely determined by the aggregate of sequences $\left(f^{\prime}\left(x_{p}\right)\right)_{n}$, and therefore by the aggregate of associated series $\sum_{0} t_{p}^{n} f_{n}\left(x_{p}\right)$. In other words when the general term in each series of the aggregate is known, so also is the general term $t_{n} f_{n}(x)$, and evidently this term can be found by multiplying together formally all the series in the aggregate, or as we shall write it symbolically

$$
\prod_{p}\left[\sum_{0} t_{p}^{n} f_{n}\left(x_{p}\right)\right]=\sum_{1} t_{n} f_{n}(x),
$$


for the coefficient of $t_{n}=t_{\alpha}^{a} t_{\beta}^{b} \cdots t_{\gamma}^{c}$ in the formal product is $f_{a}\left(x_{\alpha}\right) f_{b}\left(x_{\beta}\right)$ $\cdots f_{c}\left(x_{\gamma}\right)=f_{n}(x)$. This is the formal equivalent of $E$ composition.

7. Generators. 'These play a part in $E$ analogous to that of logarithms in common arithmetic. In the typical factor of the above formal product write $t_{p} \equiv t, x_{p} \equiv \xi$, so that it becomes $F(t, \xi) \equiv \sum_{0} t^{n} f_{n}(\xi)$, which is to be regarded as a function of two independent variables $t, \xi$. We define $F(t, \xi)$ to be the generator of $f_{n}(x)$, and write

$$
F(t, \xi) \cdot \Gamma \cdot f_{n}(x),
$$

which is to be read: $F(t, \xi)$ is the generator of $f_{n}(x)$.

To write down the $f_{n}(x)$ generated by $F(t, \xi) \equiv \sum_{0} t^{n} f_{n}(\xi)$ we first set $f_{n}(x)$ $=f_{a}\left(x_{\alpha}\right) f_{b}\left(x_{\beta}\right) \cdots f_{c}\left(x_{\gamma}\right)$, where $n=\alpha^{a} \beta^{b} \cdots \gamma^{e}$ is the prime factor resolution of $n$, and then in this substitute for $f_{a}\left(x_{a}\right), f_{b}\left(x_{\beta}\right), \cdots, f_{c}\left(x_{\gamma}\right)$ as defined by $f_{n}(x)$ which is given, since $F(t, \xi)$ is assumed known. Thus $f_{a}\left(x_{a}\right)$ is obtained from $f_{n}(\xi)$ by putting $n=a, \xi=x_{\alpha}$. E. g., if $f_{n}(\xi)=\xi^{n}, F(t, \xi) \equiv \sum_{0} t^{n} \xi^{n}$ generates $x_{\alpha}^{a} x_{\beta}^{b} \cdots x_{\gamma}^{c}=x_{n}$. Conversely, to write down the generator when the form of $f_{n}(x)$ is known, $=f_{a}\left(x_{\alpha}\right) f_{b}\left(x_{\beta}\right) \cdots f_{c}\left(x_{\gamma}\right)$, we replace in any factor, say $f_{a}\left(x_{\alpha}\right)$, the argument $x_{\alpha}$ by $\xi$ and the suffix by $n$, getting $f_{n}(\xi)$, multiply by $t^{n}$ and sum for $n=0,1,2, \cdots:$ thus $\sum_{0} t^{n} f_{n}(\xi),=F(t, \xi)$.

Either from our definitions or by convention $t^{0}$ has with respect to powers of $t$ the multiplicative properties of unity, and similarly for each of the identical symbols $f_{0}(\xi), g_{0}(\xi), \cdots$ with respect to each of $f_{n}(\xi), g_{n}(\xi), \cdots$. Only these properties will be used in generators, so that each of these symbols may be replaced by 1. A generator therefore is a power series in $t$ whose first term is 1 and in which the coefficients of the several powers of $t$ are functions of $\xi$ (some or all of which may reduce to constants). With each generator is associated ( $\$ 1(9.1))$ a sequence, and these sequences are combined only according to $C$ multiplication and division; with the sequences resulting from these operations are associated series, and clearly each of these series is a generator. Hence, under $C$, multiplication (and division) of series generators form a group.

Algebra $E$ is concerned with the relations between the $f_{n}(x), g_{n}(x), \cdots$ generated by the members of this group. Specific applications of $E$ are determined by special choices of the initial generators (e. g., each $f(\xi), g(\xi), \cdots$ is an algebraic function of $\xi$ ), and specific interpretations of the system of elements $x_{n}$ upon which the $f, g, \cdots$ are constructed (e.g., $x_{n}=n$, giving ordinary arithmetic). By means of the Maclaurin development after Theorem II" $^{\prime \prime}$ it may or may not be possible in a given application to reduce all of the generators in the group to finite form. This is the case, however, in one of the most important applications (§12), when generators are combined according to the rules for the multiplication of ordinary algebraic fractions in two letters. 
Understanding that the arguments are $t$, $\xi$ we may shorten $F(t, \xi)$ to $F$, and similarly $f_{n}(x)$ may be abbreviated to $f$, so that the generational relation will be written where convenient as $\boldsymbol{F} \cdot \boldsymbol{\Gamma} \cdot f$.

8. The definition (15) and $\S 5$ yield an important consequence. Take the $C$ product, (§2), $\left(f\left(x_{p}\right), g\left(x_{p}\right), \cdots, h\left(x_{p}\right)\right)_{n}$, of the primary functional sequences $\left(f\left(x_{p}\right)\right)_{n},\left(g\left(x_{p}\right)\right)_{n}, \cdots,\left(h\left(x_{p}\right)\right)_{n}$, noting that the argument $x_{p}$, and hence the prime $p>1$, is the same in all. If for a moment we denote the $n$th element of this $C$ product by $\varphi_{n}\left(x_{p}\right), \equiv{ }_{n}\left(f\left(x_{p}\right), g\left(x_{p}\right), \cdots, h\left(x_{p}\right)\right)$ we have $\left(\varphi\left(x_{p}\right)\right)_{n}$ a primary functional sequence, and we can form the $E$ composite $\left[\left(\varphi\left(x_{p}\right)\right)_{n}\right] \equiv\left[\left(f\left(x_{p}\right), g\left(x_{p}\right), \cdots, h\left(x_{p}\right)\right)_{n}\right]$. On the other hand we form the $D$ product, $(\S 3),\{f(x), g(x), \cdots, h(x)\}_{n}$ of the derived functional sequences $\{f(x)\}_{n},\{g(x)\}_{n}, \cdots,\{h(x)\}_{n}$, and have at once the following:

THEOREM V. The $E$ composite and the $D$ product are identical:

$$
\left[\left(f\left(x_{p}\right), g\left(x_{p}\right), \cdots, h\left(x_{p}\right)\right)_{n}\right]=\{f(x), g(x), \cdots, h(x)\}_{n} .
$$

The content of this theorem will be evident from its equivalent in generators, given next. From now on the algebra may be developed either from the standpoint of sequences or from that of generators. The latter leading at once to the extremely simple working rules of $E$, we choose it.

9. $E$ multiplication. Consider the $r$ relations

$$
F \cdot \Gamma \cdot f, \quad G \cdot \Gamma \cdot g, \quad \cdots, \quad H \cdot \Gamma \cdot h .
$$

The product $K \equiv F G \cdots H$ is a generator, say $K \cdot \Gamma \cdot k$. We define $k$ to be the $E$ product $f g \cdots h$ of $f, g, \cdots, h$. Or writing this in full, $k_{n}(x) \equiv(f g \cdots h)_{n}(x)$. That is, the generator of the $E$ product $f g \cdots h$ of $f, g, \cdots, h$ is the product of the several generators. This merely defines the symbol $f g \cdots h$ which must now be evaluated. Evidently $k_{n}(x)$ is the $n$th term ${ }_{n}\{f(x), g(x), \cdots, h(x)\}$ of the $D$ product of the $r$ sequences $\{f(x)\}_{n},\{g(x)\}_{n}, \cdots,\{h(x)\}_{n}$. Hence the explicit form of $k_{n}(x)$ is $f g \cdots h \equiv(f g \cdots h)_{n}(x) \equiv \sum_{n} f_{d}(x) g_{\delta}(x) \cdots$ $h_{\tau}(x)$, where the summation refers to all sets $(d, \delta, \cdots, \tau)$ of $r$ integers each $>0$ whose product is $n$.

The detailed proof is the same for any number of factors: let $r=2$. The $C$ product $K(t, \xi)$ of $F(t, \xi) \equiv \sum_{0} t^{n} f_{n}(\xi)$ and $G(t, \xi) \equiv \sum_{0} t^{n} g_{n}(\xi)$ is $\sum_{0} t^{n} k_{n}(\xi)$, where $k_{n}(\xi)=\sum_{r=0}^{n} f_{r}(\xi) g_{n-r}(\xi)$; and by the definition of a generator, $K(t, \xi) \cdot \Gamma \cdot k_{n}(x)$, where, $n=a^{\bullet} \beta^{b} \cdots \cdot \gamma^{\natural}$ being the prime factor resolution of $n, k_{n}(x)=k_{a}\left(x_{\alpha}\right) k_{b}\left(x_{\beta}\right) \cdots k_{c}\left(x_{\gamma}\right)$. It is to be shown that $k_{n}(x)={ }_{n}\{f(x), g(x)\}$. Let $n=d \delta$ be the resolution of $n$ into any pair of conjugate divisors $>0$, and $l=a^{a_{1}} \beta^{b_{1}} \cdots \gamma^{c_{1}}, \delta=a^{\alpha-a_{1}} \beta^{b-b_{1}} \cdots \gamma^{c-c_{1}}$ the prime factor resolutions of $d, \delta$. 
In these some of the exponents may be zeros. Substituting in $k_{n}(x)$ the values of $k_{a}\left(x_{a}\right)$, $k_{b}\left(x_{\beta}\right), \cdots, k_{c}\left(x_{\gamma}\right)$ defined by the generator $K(t, \xi)$ we get for $k_{n}(x)$ the expression

$$
\begin{aligned}
{\left[\sum_{a_{1}=0}^{\infty} f_{a_{1}}\left(x_{\alpha_{\alpha}}\right) g_{a-a_{1}}\left(x_{\alpha}\right)\right][} & \left.\sum_{b_{1}=0}^{b} f_{b_{1}}\left(x_{\beta}\right) g_{b-b_{1}}\left(x_{\beta}\right)\right] \cdots\left[\sum_{a_{1}=0}^{c} f_{c_{1}}\left(x_{\gamma}\right) g_{c-c_{1}}\left(x_{\gamma}\right)\right] \\
& =\sum f_{a_{1}}\left(x_{a}\right) f_{b_{1}}\left(x_{\beta}\right) \cdots f_{c_{1}}\left(x_{\gamma}\right) g_{a-a_{1}}\left(x_{\alpha}\right) g_{b-b_{1}}\left(x_{\beta}\right) \cdots g_{c-c_{1}}\left(x_{\gamma}\right),
\end{aligned}
$$

the $\sum$ referring to all $a_{1}, b_{1}, \cdots, c_{1}$ defined by $0 \leqq a_{1} \leqq a, 0 \leqq b_{1} \leqq b, \cdots, 0 \leqq c_{1} \leqq c$. But

$$
f_{a_{1}}\left(x_{\alpha}\right) f_{b_{1}}\left(x_{\beta}\right) \cdots f_{c_{1}}\left(x_{\gamma}\right)=f_{d}(x), \quad g_{a-a_{1}}\left(x_{\alpha}\right) g_{b-b_{1}}\left(x_{\beta}\right) \cdots g_{c-c_{1}}\left(x_{\gamma}\right)=g_{\delta}(x) .
$$

Hence $k_{n}(x)=\sum_{n} f_{d}(x) g_{\delta}(x)$, the sum referring to all pairs of divisors $d, \delta>0$ of $n$ such that $d \delta=n$. That is, $k_{n}(x)={ }_{n}\{f(x), g(x)\}$.

Obviously $f g \ldots h$ is invariant under all permutations of $f, g, \cdots, h$; hence $F$ multiplication is commutative. That it is also associative follows immediately as in $D$.

(16) The unit of $E$ multiplication is $\eta \equiv \eta_{n}(x) \equiv \eta_{x}(n)$ defined by $\eta_{x}(1)=1$, $\eta_{x}(n)=0(n>1)$.

10. $E$ division. By definition the $E$ reciprocal $f^{\prime}$ of $f$ is generated by the reciprocal $F^{\prime}$ of the generator $F$ of $f$. In this $F^{\prime}$ is obtained by the formal division of 1 by $F$. Since $F F^{\prime}=1, F F^{\prime}$ is the generator of $\eta$.

With respect to $E$ multiplication and division $\eta$ has the properties of unity in ordinary algebraic multiplication and division, for $\eta f=f$, so that the factor $\eta$ may be omitted from any $E$ product, and evidently $\eta$ is its own reciprocal. Since $F$ is any generator, $\eta$ has an infinity of generators. By an obvious algebraic analogy, $f^{\prime}=\eta$ may be written when convenient in either of the forms $f=\eta / f^{\prime}, f^{\prime}=\eta / f$.

The $E$ reciprocal of an $E$ product is obviously the $E$ product of the several $E$ factors of the product:

$$
\frac{\eta}{f g \cdots h}=\frac{\eta}{f^{\prime}} \frac{\eta}{g} \cdots \frac{\eta}{h}
$$

The $E$ product of $f g \ldots h$ and $\eta / \varphi \psi \cdots \chi$ is written in the form

$$
\frac{f g \cdots h}{\varphi \psi \cdots \cdot \chi}
$$

and clearly such $E$ fractions have all the multiplicative properties of ordinary fractions. 
11. $E$ addition and subtraction. Since by $\$ 4(14)$ (end) the $f_{n}(x)$, $g_{n}(x), \ldots$ form an annulus, $\pm f_{n}(x) \pm g_{n}(x) \pm \ldots$, for a definite choice of the signs in an element $l_{n}(x)$ of the annulus. Shortening the notation as before we write, for a definite choice of the signs $l \equiv \pm f \pm g \cdots$, thus defining $E$ addition and subtraction. For the same choice of signs the $E$ product of $\varphi$ and $l$ is $\varphi l$, and at once from the definitions we have

$$
\varphi l= \pm \varphi f \pm \varphi g \pm \cdots
$$

Since $E$ addition and subtraction are obviously associative these operations have all the formal properties of algebraic addition and subtraction.

In full for $l=f+g$ : let $l_{n}(x)=f_{n}(x)+g_{n}(x)$. By the definition of $E$ multiplication, $(\varphi l)_{n}(x)=\sum_{n} \varphi_{d}(x) l_{\delta}(x)$, the sum extending to all pairs of integers $d, \delta>0$ such that $d \delta=n$. Hence $(\varphi l)_{n}(x)=\sum_{n} \varphi_{d}(x)\left[f_{\delta}(x)+g_{\delta}(x)\right]=\sum_{n} \varphi_{d}(x) f_{\delta}(x)+\sum_{n} \varphi_{d}(x) g_{\delta}(x)$ $=(\varphi f)_{n}(x)+(\varphi g)_{n}(x)$; viz., $\varphi l=\varphi f+\varphi g$.

THEOREM VI. The aggregate of all the elements of all derived functional sequences forms a system with respect to the four $E$ rational operations.

Fractional and negative powers are introduced precisely as in $C$, and combining these with Theorem VI, we have the complete algebra $E$.

We note that the product $f_{n}(x) g_{n}(x) \cdots h_{n}(x)$ is an element of the above aggregate. It cannot be abbreviated to $f g \cdots h$ in applying $E$, for this denotes the $E$ product. We accordingly use $|f g \cdots h|$, writing therefore

$$
|f g \cdots h|_{n}(x) \equiv f_{n}(x) g_{n}(x) \cdots h_{n}(x) .
$$

With this we form $E$ products. Thus the $E$ product of $|f g|$ and $k$ is written $|f g| k$, and is in full $\sum_{n} f_{d}(x) g_{d}(x) k_{\delta}(x)$.

The algebra is used as outlined in the following example.*

12. Example. Let

$$
F(t, \xi) \equiv 1+t f_{1}(\xi)+\cdots+t^{n} f_{n}(\xi)
$$

be a polynomial in the two independent variables $t, \xi$ of degree $n>0$ in $t$, and let all the coefficients of $F$ lie in the domain of rationality $\nabla$. We call $F$

* Detailed applications of this have been made with numerous special theorems as examples, by Miss E. D. Pepper, Tôhoku Mathematical Journal, vol. 22, Nos. 1, 2. The theorems include many of those mentioned at the end of $\S 1$ and are sufficient in number to show the increase in power and the great saving in space and labor of manipulation gained by these methods. Finally the paper contains applications of $E$ to the multiplicative properties of algebraic numbers, the ideals of any realm, and systems of polynomials to a single or a double modulus. It will therefore be sufficient here to sketch the development in one case. 
and $1 / F$ primary generators, which are prime or composite according as $F$ is irreducible or reducible in $\nabla$. The aggregate $P$ of all primary generators obtained by taking $n=1,2, \cdots$, and $f_{1}(\xi), f_{2}(\xi), \cdots$ successively equal to all polynomials in one variable $\xi$ with coefficients in $\nabla$ may be arranged in pairs $\left(F, F^{\prime}\right),\left(G, G^{\prime}\right), \cdots$, such that either generator in a pair is the reciprocal of the other. If $F \cdot \Gamma \cdot f, F^{\prime} \cdot \Gamma \cdot f^{\prime}$, then since $f^{\prime}=\eta / f, f=\eta / f^{\prime}$, we may take as fundamental either $f$ or $f^{\prime}$, regarding the other as its reciprocal. We shall choose as fundamental that one of $f, f^{\prime}$ whose generator is a polynomial, viz., is in finite form. For example, of $f^{\prime}, f^{\prime \prime}$ generated by $1-t \xi$ and $\sum_{0} t^{n} \xi^{n}$ respectively, $f$ is fundamental. The generator $F$ of a fundamental $f$ is called fundamental, and $f$ is prime or composite (in $\nabla$ ) according as $F$ is prime or composite.

If in the $E$ product $f g \ldots h$ the $r$ factors $f, g, \cdots, h$ are identical, it is written $f^{r}$, and by convention $f^{0}=\eta$. Obviously if $F \cdot \Gamma \cdot f$, then $F^{r} \cdot \Gamma \cdot f^{r}$ and $F^{\prime r} \cdot \Gamma \cdot f^{\prime}$ where $F^{\prime}=1 / F, f^{\prime}=\eta^{\prime} f$. Any fundamental generator may be resolved uniquely in $\nabla$ into a product of powers of distinct prime fundamental generators. Let $F=G^{a} H^{b} \ldots K^{c}$ be the prime resolution of $F$, and let $F \cdot \Gamma \cdot f, G \cdot \Gamma \cdot g$, etc. Then $f^{\prime}=g^{a} h^{b} \cdots k^{c}$ is called the prime resolution of $f$ (in $\nabla$ ), and similarly for $f^{\prime}=\eta / f=\eta / g^{a} h^{b} \cdots k^{c}$. These resolutions are unique.

Next consider the aggregate $\boldsymbol{\Pi}$ of all generators formed from the products $1,2,3, \cdots$ at a time of all the generators in $P$. Any generator in $\Pi$ is of the form $F / \Phi$; where $F, \Phi$ are primary and fundamental, so that $F, \Phi$ are in finite form and are not resolved into their factors in $\nabla$. If $F, \Phi$ are relatively prime in $\nabla, F / \Phi$ is called reduced, and likewise for $f / \varphi$ where $F / \Phi \cdot \Gamma \cdot f / \varphi$. If in this case $f=g^{a} h^{b} \cdots k^{c}, \varphi=\lambda^{m} \mu^{n} \cdots \varrho^{s}$ are the prime resolutions of $f, \varphi$, we call $g^{a} h^{b} \cdots k^{c} / \lambda^{m} \mu^{n} \cdots \varrho^{s}$ the prime resolution of $f / \varphi$. If $F=T F_{1}$, $\boldsymbol{\Phi}=T \Phi_{1}$ where $F_{1}, \Phi_{1}$ are relatively prime in $\nabla, F / \Phi=F_{1} / \Phi_{1}$ and the latter, being reduced, yields the prime resolution of $f / \varphi$. Or the same result can be reached by cancellation of powers of $g, h, \cdots$ against like powers of identical $\lambda, \mu, \cdots$ in $f / \varphi$ after the prime resolutions of $f, \varphi$. From the manner in which it has been obtained the prime resolution of $f / \varphi$ is unique.

The aggregate of all $E$ quotients $f / \varphi$, including all cases in which either $f$ or $\varphi$ is the unit $\eta$, is denoted by $A$; the generators of these quotients are those in $\Pi$.

To investigate the relation between given members of $A$ we first find their prime resolutions and then note the multiplicative relations between the results to obtain identities, performing multiplications and divisions as in arithmetic. Two problems arise which are complementary to one another. First, suppose we are given the generatol, which may be of one of the forms 
$F, 1 / F, F / G$, where $F, G$ are fundamental and primary; it is required to find the corresponding $f, \eta / f, f / g$. The most general form of $F$ is

$$
F(t, \xi) \equiv 1+t^{a_{1}} f_{a_{1}}(\xi)+\cdots+t^{a} \cdot f_{a_{0}}(\xi),
$$

where none of the polynomials $f_{j}(\xi)$ with coefficients in $\nabla$ is identically zero, and the $a$ 's are integers $>0$. There is no difficulty in writing down the $f_{n}(x)$ generated by this (cf. $\S 7$ ), but as the expression is complicated when $s>1$, we omit it. In the forms $1 / F, F / G$ we first perform the formal divisions and then proceed as in $\$ 7$. Next, if we are given the $f_{n}(x)$ we find its generator by $\$ 7$. If this generator is in $A$, it is a matter of algebra to reduce it to finite form and find its resolution in $\nabla$ into prime factors, whence, noting the functions generated by the several prime generators, we get the prime resolution of $f$. The identities between the $f, g, \cdots$ may be written down either from the generators or the prime resolutions. Each such identity gives a result of the kind mentioned in the introduction (a simple example is given at the end of the paper). The same identity may be read in many ways; thus $f^{2} g^{2} h=f g h \cdot g h=f^{2} \cdot g^{2} \cdot h$, etc., where in $f g h \cdot g h$ we take first the $E$ products $f g h, g h$ and then take the $E$ product of the results. Each such reading gives a theorem.

To get theorems relating to the natural numbers take $x_{p}=p$ ( $p$ any prime), and hence $x_{n}=n(n$ any integer $>0)$. This is called the numerical case. It refers to the arithmetical functions built up from the concept of divisibility and the unique factorization law, sometimes called numerical functions.

The aggregate $I$ is characterized by two essential features: (1) each generator in $\boldsymbol{I}$ is expressible in finite form as a rational algebraic function of two variables; (2) the coefficients of each generator are in $\nabla$. An examination of Dickson's History (loc. cit.) will show that all of the numerical functions in the literature have simple generators satisfying (1), and that the majority satisfy also (2). The only exceptions are those in which occur, as coefficients of the several powers of $t$, instead of algebraic functions of $\xi$, certain simple transcendental functions, such as $(-1)^{\xi}$, or those expressing a quadratic character. Moreover with one exception $\nabla \equiv R(1)$, the rational domain. In the exception $\nabla=R(i \sqrt{3})$.

Even trivial algebraic identities yield results of interest. Thus it is readily seen that in the numerical case the equivalent of

$$
\frac{1}{1-t} \cdot \frac{1-t}{1-t \xi}=\frac{1}{1-t \xi}
$$

is the totient theorem cited in $\S 1$. Of this kind of theorem some of Gegenbauer's are as complicated as any in the literature.* For example, if $J_{\nu}(n)$ is Jordan's generalization of Euler's

* Cf. Dickson, History, vol. 1, p. 298 (72). 
$\varphi(n), J_{\nu}(n)=$ the number of different sets of $\nu$ equal or distinct positive integers $\leqq n$ whose G. C. D. is prime to $n$, and $u_{k}(n)=n^{k}$, and $\rho_{k, g}(n)=$ the sum of the $k$ th powers of those divisors $d_{\text {, of }} n$ whose conjugate divisors are exact sth powers, Gegenbauer gives

$$
\sum_{n} J_{\nu}(d) d^{k} \rho_{k, e}\left(\frac{n}{d}\right)=\rho_{k+\nu, d}(n)
$$

which in $E$ notation is $\left|J_{\nu} u_{k}\right| \rho_{k, e}=\rho_{k+\nu, e}$. From the abore definitions it is easily seen that

$$
\frac{1-\xi^{k} t}{1-\xi^{k+\nu} t} \cdot I \cdot\left|\cdot J_{\nu} u_{k}\right|, \quad \frac{1}{\left(1-\xi^{k} t\right)\left(1-t^{*}\right)} \cdot I^{\prime} \cdot \rho_{k, e} .
$$

Hence ('egenbauer's result is the translation of the identity

$$
\frac{1-\xi^{k} t}{1-\xi^{k+\nu} t} \cdot \frac{1}{\left(1-\xi^{k} t\right)\left(1-t^{2}\right)}=\frac{1}{\left(1-\xi^{k+\nu} t\right)\left(1-t^{2}\right)} \text {. }
$$

Noting that $\lambda(n) \equiv+1$ or -1 according as the total number of prime divisors of $n$ is cren or odd is generated by $1 /(1+t)$, and that Möbius's $\mu(n)$ is generated by $(1-t)$, also $\prime^{2}$ by $(1+t)$, and that the generator of $\sigma_{k}(n)-:$ the sum of the $k$ th powers of all the divisors of $n$ is $1:\left(1-\xi^{k} t\right)$, also that $1 /\left(1-t^{*}\right)$ generates $\tau_{s}(n) \equiv 1$ or 0 accoriling as $n$ is or is not the sth power of an integer $>0$, and that $1-\xi^{k} t$ generates $\left|\mu u_{k}\right|$, we see that the same simple identity yields several more results upon rearrangement of its factors, etc., either for special values of $k, \nu, s$ or for general.

UNIVERSITY OF IVASHINGTON, SEATTLE, WaSh. 